\title{
An autosomal locus causing autoimmune disease: autoimmune polyglandular disease type I assigned to chromosome 21
}

\author{
Johanna Aaltonen ${ }^{1}$, Petra Björses ${ }^{1}$, Lodewijk Sandkuijl ${ }^{3}$, Jaakko Perheentupa ${ }^{2}$ \\ \& Leena Peltonen ${ }^{1}$
}

\begin{abstract}
Autoimmune polyglandular disease type I (APECED) is an autosomal recessive autoimmune disease characterized by a variable combination of the failure of the endocrine glands. The pathogenesis of this unique autoimmune disease is unknown; unlike many other autoimmune diseases, APECED does not show association to specific HLA haplotypes. Unravelling the APECED locus will identify a novel gene outside the HLA loci influencing the outcome of autoimmune diseases. We have assigned the disease locus to chromosome 21 q22.3 by linkage analyses in 14 Finnish families. Linkage disequilibrium studies have significantly increased the informativeness of the analyses and helped to locate the critical DNA region for the APECED locus to just 500 kilobases, a much more precise definition than linkage analyses alone could achieve.
\end{abstract}

${ }^{1}$ Department of

Human Molecular

Genetics, National

Public Health

Institute,

Mannerheimintie

166, FIN-00300

Helsinki, Finland

${ }^{2}$ Children's

Hospital,

Stenbäckinkatu 11,

FIN-00290

Helsinki, Finland

${ }^{3}$ Institute of Clinical

Genetics

Erasmus University, PO Box 1738, 3000 $D R$

Rotterdam, The Netherlands, and

Department of

Human Genetics,

Sylvius

Laboratories,

Wassenaarseweg 72 ,

2333 AL, Leiden,

The Netherlands

Correspondence should be addressed to L.P.
Genetic factors are known to influence susceptibility to many autoimmune diseases, but their exact nature remains unknown ${ }^{1,2}$. Unravelling the molecular pathogenesis of inherited autoimmune diseases could serve as a shortcut to the dissection of the individual components in the molecular background of an abnormal immune response.

Autoimmune polyglandular disease type I (APDI), also known as autoimmune polyendocrinopathy-candidiasisectodermal dystrophy (APECED), is an autosomal recessive disease (MIM no. 240300) resulting in a variable combination of failure of the parathyroid glands, adrenal cortex, gonads, pancreatic $\beta$ cells, thyroid gland and gastric parietal cells $s^{3-6}$. Other features include alopecia, vitiligo, hepatitis, chronic mucocutaneous candidiasis, dystrophy of the dental enamel and nails and keratopathy. A few cases of squamous cell carcinoma of the oral mucosa have also been reported among APECED patients ${ }^{6}$. The disease usually manifests itself in childhood, but new tissue specific symptoms may appear throughout life. The incidence of APECED in Finland is approximately $1: 25$, 000 , the population demonstrating an exceptionally high disease prevalence ${ }^{5}$. The disease is also found at a very high frequency among Iranian Jews ${ }^{7}$.

The population of Finland exhibits many consequences of the founder effect and genetic isolation; mutations causing several recessive diseases, rare elsewhere in the world, are enriched in this population ${ }^{8}$. Each of the diseasecausing alleles is probably inherited from one member of a relatively limited founder population which arrived in Finland some 1,500-2,000 years ago. The assumption of one major mutation in Finnish diseases is based on population history and has proved to be true when the actual molecular defect has been identified, for example, in aspartylglucosaminuria (AGU), in which one mutation is found in $98 \%$ of Finnish disease alleles ${ }^{9}$. In such an isolated population linkage disequilibrium and allelic association studies are particularly useful and help to define the DNA region of the disease gene of interest with greater accuracy than would be possible bylinkage analysis alone $\mathrm{e}^{10,11}$.

Here we have taken advantage of these special features of the Finnish population to identify an autosomal locus behind an inherited autoimmune disease. Although APECED is a rare disease, it represents a unique biological model for human autoimmunity and identification of its gene defect should provide new insights into the abnormalities of immune responses in general.

\section{Linkage analyses}

To search for the APECED locus, we tested 250 amplifiable markers spread randomly at $\sim 20 \mathrm{cM}$ intervals among all autosomes. We excluded most of the human genome in our family material ${ }^{12}$ (Fig. 1) before highly significant lod scores were observed with markers localized to the long arm of chromosome 21.

Due to the relatively late appearance of symptoms in some patients, we adopted age-dependent penetrance classes in the initial analysis (see Methodology). Furthermore, as it is possible that some cases will remain non-diagnosized due to the relatively mild features of the disease phenotype such as candidiasis, we adopted a $95 \%$ maximal penetrance.

The lod scores obtained in pairwise linkage analyses between APECED and markers located on 21q22.3 are shown in Table 1. The best pairwise lod scores were obtained with D21S49. We based our analysis on the most 

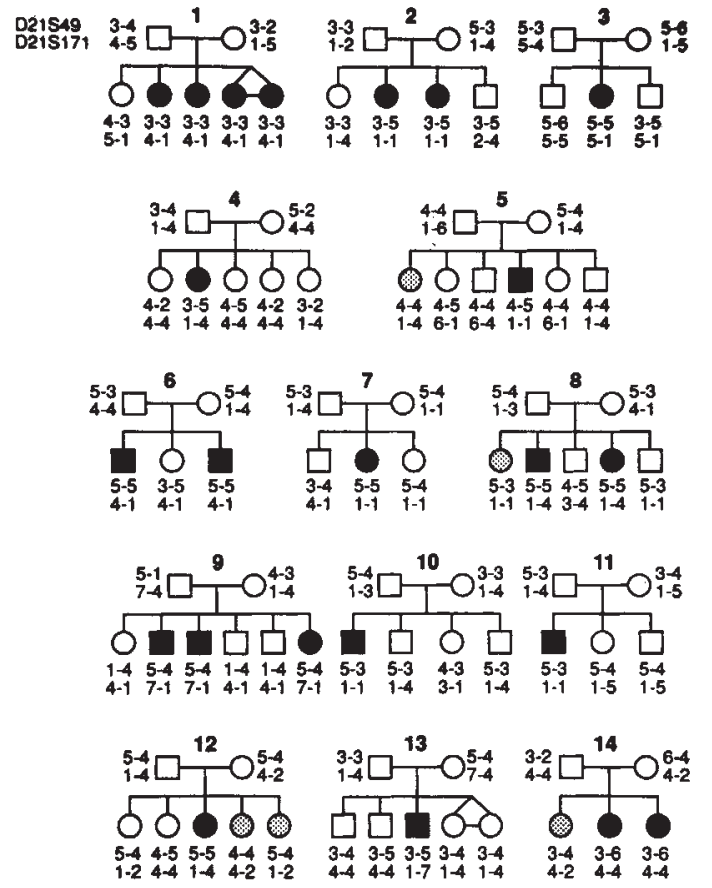

recently published integration map of this chromosomal region ${ }^{13}$. For markers D21S416, D21S1224, D21S1235 and D21S1225 no published mapping data was available and we estimated the relative position of these closely linked markers by linkage analysis using the ILINK program in our family series (Fig. 2). No obligatory recombination events were observed between the disease and either $D 21 S 49$ or $P F K I$, whereas D21S171 and D21S1225revealed one and two recombination events respectively. D21S49 was uninformative in the two families which demonstrated
Fig. 1 The APECED pedigrees and observed alleles for markers D21S49 and D21S171 showing significant allelic association. The shadowed individuals indicate uncertain diagnoses.

recombinations between the disease and D21S1225. Based on these recombinations, the APECED locus was localized to the $2.6 \mathrm{cM}$ chromosomal region between D21S1225 and D21S171.

Multipoint LINKMAP analysis was performed without adopting the age-dependent penetrance classes as they did not have a significant effect on the result obtained by pairwise analysis. Using LINKMAP, information from markers D21S212, D21S1225, D21S49, PFKL and D21S171 was combined and a maximal positional lod score of 8.14 was obtained. Multipoint analysis assigned the disease locus between $D 21 S 49$ and $D 21 S 171$ and the peak value of the lod score was obtained between markers PFKL and D21S171 (Fig. 3).

\section{Linkage disequilibrium}

In isolated populations with a high probability of one common ancestral mutation, linkage disequilibrium is a very useful method for positioning the disease locus with greater accuracy. We tested for possible linkage disequilibrium with the two closely linked markers using a $\chi^{2}$ test. D21S49 and D21S171 showed significant linkage disequilibrium $(p<0.001)$. The alleles observed in our family series are shown in Table 2 . With D21S49, allele 5 was found in $61 \%$ of APECED chromosomes and allele 1 of $D 21 S 171$ was found in $64 \%$ of disease chromosomes, as opposed to $0 \%$ and $7 \%$, respectively, in non-APECED (control) chromosomes. Analysis of haplotypes generated with D21S49 and D21S171 revealed eight different haplotypes in APECED chromosomes and 13 different haplotypes in non-APECED chromosomes. Haplotype 5-1 was the most common on APECED chromosomes at $36 \%(0 \%$ in controls); 3-1 was observed in $21 \%$ of APECED chromosomes (4\% in controls) and 5-4 in 14\% of APECED chromosomes ( $0 \%$ in controls) (Fig. 1).

When the observed linkage disequilibrium was applied to linkage analyses, the significance of the obtained lod scores increased (Table 1 ): in the case of D21S49, for example, from 6.49 to 12.59 . We also used linkage disequilibrium in the multipoint linkage analysis in which we combined information from D21S1225, D21S49 and D21S171 (dashed line in Fig. 3). The highest positional lod score was again obtained between D21S49 and D21S171, but now with a lod score of 15.16 compared to the earlier lod score of 8.14. The best positional lod score also shifted slightly towards a more centromeric position, placing APECED closer to D21S49. 
Fig. 2 The order and inter-marker distances ${ }^{13}$ of the markers used in linkage analysis on chromosome 21q22.3.

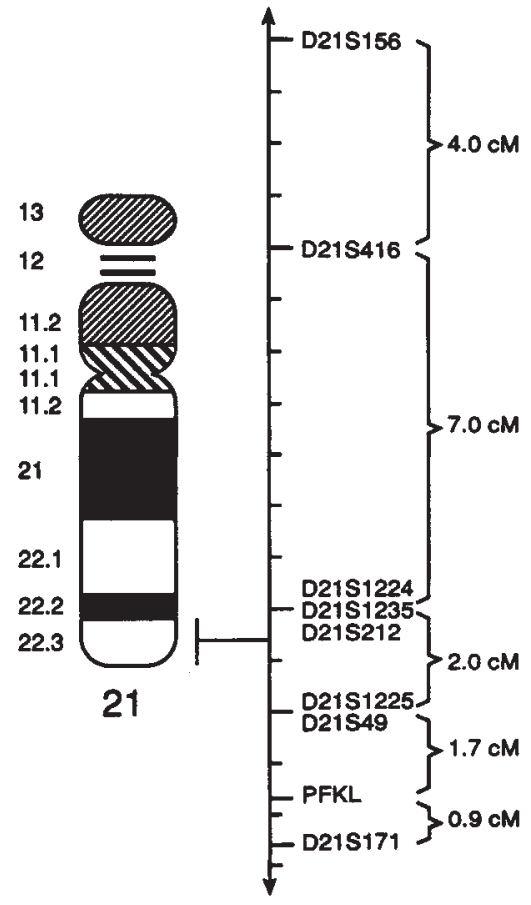

\section{Luria Delbrück analysis}

To define further the critical DNA region of the APECED locus, we applied Luria and Delbrück's formula to estimate the physical distance between the gene and markers demonstrating the most significant linkage disequilibrium ${ }^{14}$. This formula was originally developed for estimating mutation rates in rapidly growing bacterial populations, but has recently been applied to evaluate distances between disease loci and markers in diseases enriched in isolated populations ${ }^{10,11}$. If one assumes that there is a single ancestral mutation segregating in the Finnish population over 100 generations, the Luria Delbrück analysis suggests a recombination frequency of 0.0045 between $A P E C E D$ and D21S49 (likely range

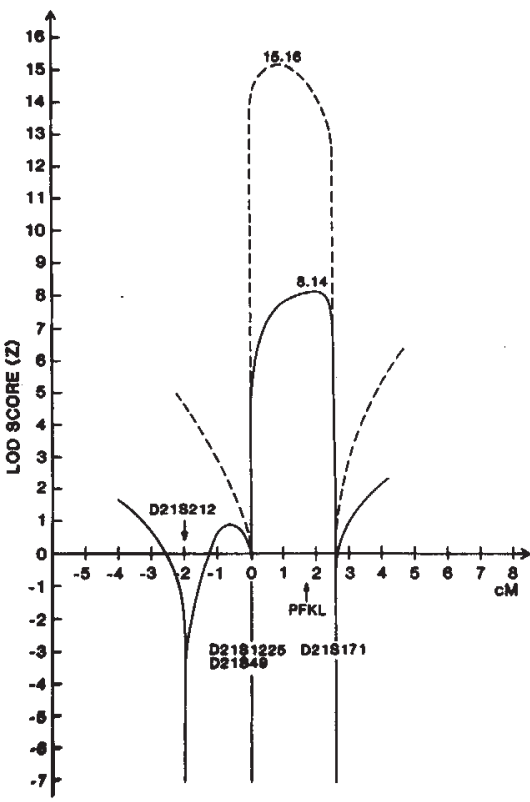

0.00368 -0.00576, according to the Luria-Delbrück equations), which corresponds to a distance of about 0.5 $\mathrm{cM}$, or $500 \mathrm{~kb}$ (assuming the average correspondence of 1 $\mathrm{cM}$ to about $1 \mathrm{Mb}$ ).

\section{Discussion}

Genetic studies of experimental models of autoimmunity have revealed the importance of the HLA region on chromosome 6 and further suggested that there must be several genes outside the HLA region that play a role in the development of autoimmunity ${ }^{15}$. However, so far no single gene has been shown to be responsible for the initiation and development of human autoimmune disease. We have used enrichment of an inherited autoimmune disease, APECED, in the Finnish population to assign the disease locus to chromosome $21 \mathrm{q} 22.3$ using linkage analyses in 14 families. The random mapping approach in this clinically heterogeneous disease is especially problematic and we took several precautions performing the linkage analyses. We used highly conservative diagnostic criteria to avoid false diagnosis but phenocopies were not allowed. It is difficult to make the clinical diagnosis with certainty in a few borderline cases, but the disease is so typical once those borderline cases are omitted that one would not expect any phenocopies among the definite cases. We also adopted age-dependent penetrance classes for healthy individuals, as the disease may appear later in life and maximal $95 \%$ penetrance for the disease gene was allowed to avoid missclassification of family members with subclinical phenotype. These precautions resulted clearly in some loss of power in the linkage analyses in this limited family material.

The fact that APECED is enriched in the Finnish population allowed the use of linkage disequilibrium analysis to refine the disease locus. One can assume that the disease is caused by one major ancient mutation from a common ancestor in the founder population. We have demonstrated previously that this is the case in another relatively common Finnish disease, aspartylglucosaminuria'. Consequently, affected chromosomes descending from a common ancestral mutation should carry a similar distinctive haplotype in the immediate vicinity of the gene; our preliminary haplotype data on APECED chromosomes actually supports this assumption. When we adopted the linkage disequilibrium observed with two markers to linkage analyses, the informativeness of the analyses increased significantly (as we have shown for another Finnish disease ${ }^{16}$ ). Our results emphasize again the importance of routinely incorporating a linkage disequilibrium estimation (between the disease and specific alleles) in linkage strategies, and should encourage the use of random gene mapping even when family materials are limited.

The concept of one major founder mutation and observed linkage disequilibrium in the APECED chromosomes offers a distinct advantage in the identification of the critical chromosomal region, as

Fig. 3 Chromosome 21 location map summarizing lod scores calculated for APECED in 14 Finnish families. $D 21 S 49 / D 21 S 1225$ was arbitrarily placed at 0 . Solid line, multipoint analysis without adopting age-dependent penetrance classes assuming equilibrium; dashed line, linkage disequilibrium included in the analysis. 
Table 2 Distribution of alleles at $D 21549$ and $D 215171$ in parental chromosomes

\begin{tabular}{|c|c|c|c|c|c|}
\hline \multicolumn{3}{|c|}{$021 \$ 49$} & \multicolumn{3}{|c|}{$D 21 S 171$} \\
\hline Allele & $\begin{array}{l}\text { APECED } \\
\text { chr. }\end{array}$ & $\begin{array}{c}\text { Control } \\
\text { chr. }\end{array}$ & Allele & $\begin{array}{l}\text { APECED } \\
\text { chr. }\end{array}$ & $\begin{array}{c}\text { Contro } \\
\text { chr. }\end{array}$ \\
\hline 1 & 0 & 1 & 1 & 18 & 2 \\
\hline 2 & 0 & 3 & 2 & 0 & 3 \\
\hline 3 & 8 & 10 & 3 & 0 & 2 \\
\hline 4 & 2 & 13 & 4 & 7 & 16 \\
\hline 5 & 17 & 0 & 5 & 1 & 4 \\
\hline \multirow{2}{*}{6} & 1 & 1 & 6 & 0 & 1 \\
\hline & & & 7 & 2 & 0 \\
\hline Total & 28 & 28 & Total & 28 & 28 \\
\hline$\chi^{2}=29.2$ & \multicolumn{2}{|c|}{$p=0.00002$} & $\chi^{2}=26$ & \multicolumn{2}{|c|}{$p=0.00021$} \\
\hline
\end{tabular}

information is obtained from recombination events occurring over the entire history of a population. The disease allele was most probably introduced into the Finnish population sufficiently long ago that chromosomal recombinations have produced a small region of strong linkage disequilibrium; this seems to justify the use of Luria and Delbrück's formula to define better the chromosome region of interest ${ }^{10,11}$. Multipoint linkage analysis and observed recombinations would indicate that the APECED locus lies in an interval of $2.6 \mathrm{cM}$. When the Luria-Delbrück formula is used to estimate the distance between the disease locús and its closest marker, it places the APECED locus within $500 \mathrm{~kb}$ of D21S49, a realistic distance to be covered with molecular cloning techniques. The actual distance may be even smaller, since a fraction of the disease chromosomes that do not carry the highrisk marker allele may represent new mutations. In a similar analysis, Hästbacka et al..$^{10}$ calculated that about 5,000 Finnish chromosomes would be expected to carry new mutations, which would correspond to about $7 \%$ of APECED chromosomes.

Our estimate for the critical APECED chromosomal region should be interpreted with caution, although it neatly demonstrates the power of linkage disequilibrium in the mapping of the loci for diseases enriched in genetic isolates. Chromosomal region $21 \mathrm{q} 22.3$ is problematic as markers on this region show an exceptionally high recombination rate and genetic distances $(M)$ in the area correspond to shorter physical distances (bp) than average in the genome ${ }^{17}$. This raises problems for ordering markers and estimating inter-marker distances; it could also result in misleading approximations of the chromosomal distances when using Luria and Delbrück formula.

Chromosome 21q22.3 contains four known genes: the cystathione-b-synthase (CBS) gene ${ }^{18}$ is defective in homocystinuria; the liver subunit of phosphofructokinase (PFKL) gene mutations ${ }^{19}$ may be responsible for some forms of haemolytic anaemia and mutations in the $C D 18^{20}$ gene result in leukocyte adhesion deficiency. The fourth gene coding for $\alpha$ crystallin (CRYA1) ${ }^{21}$ has not been linked to any human disease. Most probably the APECED locus represents a novel human gene requiring a spectrum of positional cloning techniques to search for novel genes potentially underlying this autoimmune disorder.

The chromosomal assignment reported here for APECED locus is important for several reasons. First, it facilitates the development of DNA diagnostic procedures in families with this complex, multicomponent disease for which no presymptomatic or prenatal diagnostic tests are available. Presymptomatic diagnosis will help to identify affected family members so that close follow-up could be focused on those individuals who have yet to develop the disease. For the first time, heterozygote carriers of the APECED gene can be identified with a relatively high degree of reliability. Most importantly perhaps, our data provides the basis for isolation of the APECED gene. Identification and characterization of this gene will most probably reveal an essential component of the human immune system and provide new insight into immunoregulation and autoimmunity in general.

\section{Methodology}

Family material. We studied 14 Finnish APECED families with 23 affected individuals (Fig. 1). Where there were identical twins only one sibling was included in our analysis. This was done for both affected and unaffected twins even though in the latter case our approach was more conservative. We took this approach since it is uncertain if non-penetrance for APECED really is a random phenomenon. Persons with a mild manifestation of only one of the signs of APECED were classified as 'diagnosis unknown' prior to the linkage analysis. Diagnosis was based on the presence of at least two of the following: chronic mucocutaneous candidiasis, hypoparathyroidism and primary adrenocortical failure ${ }^{6}$. Earlier genealogical studies established from the population registers have revealed only a very low degree of consanguinity among APECED families ${ }^{5}$. All the patients and siblings included in this study were examined personally by J.P.

DNA-markeranalysis. Genomic DNA was extracted from $10-20 \mathrm{ml}$ blood samples in accordance with standard procedures ${ }^{22}$. Polymorphic microsatellite markers were analysed using PCR and polyacrylamide gel electrophoresis (PAGE) ${ }^{23}$. PCR was performed in a total volume of $15 \mu \mathrm{l}$ containing $12 \mathrm{ng}$ template DNA, $3 \mathrm{pmol}$ primers, $0.2 \mu \mathrm{M}$ of each nucleotide, $20 \mathrm{mM}$ TrisHCl (pH 8.8), 15 $\mathrm{mM}\left(\mathrm{NH}_{4}\right)_{2} \mathrm{SO}_{4}, 1.5 \mathrm{mM} \mathrm{MgCl}_{2}, 0.1 \%$ Tween $20,0.01 \%$ gelatin and 0.25 U Taq DNA polymerase (Promega). One of the primers was labelled at the $5^{\prime}$ end using ${ }^{32} \mathrm{P}-\gamma \mathrm{ATP}$. The PCR reactions were performed in multiwell microtitre plates for 27 cycles with denaturation at $94^{\circ} \mathrm{C}$ for $30 \mathrm{~s}$, annealing at various temperatures (depending on the primers) for $30 \mathrm{~s}$ and extension at $72^{\circ} \mathrm{C}$ for $30 \mathrm{~s}$; denaturation was for $4 \mathrm{~min}$ and final extension was for $4 \mathrm{~min}$. The amplified fragments were separated using 5\% denaturing PAGE: The markers originated from the amplifiable marker collection of the Nordic Genome Resource Center in Uppsala and Genethon (The Généthon microsatellite map catalog 1992).

Linkage analyses. Linkage analyses were carried out with the MLINK, ILINK and LINKMAP options of the LINKAGE package programs

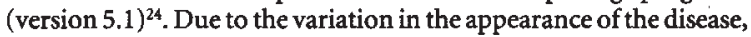
in pairwise analyses we assigned categories of $\langle 2,2-5,6-10,11-15$, $16-20,21-30,>30$ years of age and adopted penetrances of $5,20,45$, $60,70,80$ and $95 \%$, respectively, for the healthy siblings. No phenocopies were allowed in the linkage analyses. As APECED is a typical representative of Finnish disease heritage, we assumed that locus heterogeneity can be ignored. The approximate $90 \%$ confidence limits were calculated using the 11 lod down method $^{25}$. The genetic inter-marker distances used in the analysis were sex-average distances obtained from published sources ${ }^{13}$. For D21S416, D21S1224, D21S1235 and D21S1225 the locations and genetic distances to nearby markers were estimated for our family material using the ILINK option of the LINKAGE package.

Linkage disequilibrium. $\chi^{2}$ tests were carried out with the CONTING option of LINKUTIL in the LINKAGE package. In multipoint LINKMAP analyses the information from D21S1225 and D21S49 was combined and used as one marker. In LINKMAP analyses employing linkage disequilibrium, a disease gene frequency of 0.01 was used in haplotype frequencies, which were determined for each map interval depending on the location of the APECED locus. 
Luria Delbrilck analysis. Luria and Delbrück's bacterial mutation formula, which uses the observed allelic association in an isolated population, was applied for further definition of the critical DNA region for the APECED gene on the assumption that the mutation has been segregating in the Finnish population for 100 generations ${ }^{10,11,14}$. Recombination frequency was calculated from the proportion of chromosomes without the high-risk allele (allele 5 for D21S49 is not present on 11/28 disease chromosomes). It was assumed that the current population of Finland ( 5 million individuals, or $10^{7}$ chromosomes) arose from 500 founders living approximately 100 generations ago. For a constant rate of population growth of $\mathrm{e}^{\mathrm{d}}$, $\mathrm{d}$ was calculated to be 0.0921 . The frequency of the APECED gene was set equal to the square root of the incidence, which is 1 in 25,000 in Finland. Using equation (1) from Hästbacka et al. ${ }^{10}(\pi=\alpha / \mathrm{dln}(\alpha /$

Received 1 April; accepted 13 June 1994.

1. Todd, J.A. \& Steinman, L. Genetic dissection of tolerance. Curr. Op. Immunol. 4, 699-702 (1992).

2. Yetman, N., Sachs, J. \& Bottazzo, G.F. Autoimmunity - towards the year 2001. Immunol. Today 13, 239-240 (1992).

3. Ahonen, P., Koskimies, S. Lokki, M.-L., Tilikainen, A. \& Perheentupa, J. The expression of Autoimmune Polyglandular Disease Type IAppears Associated With Several HLA-A Antigens But Not With HLA-DR. J. clin. Endocrinol. With Several HLA-A Antigens
Metab. 66, 1152-1157 (1988).

4. McKusick, V.A. Mendelian Inheritance in Man 10th edn (Johns Hopkins University Press, Baltimore, 1992.)

5. Ahonen, P. Autoimmune polyendocrinopathy-candidosis-ectodermaldystrophy (APECED): Autosomal recessive inheritance. Clin. Genet. 27, 535-542 (1985)

6. Ahonen, P., Myllarniemi, S., Sipllä, I. \& Perheentupa, J. Clinical variation of autoimmune polyendocrinopathy-candidiasis-ectodermal dystrophy (APECED) in serles of 68 patients. New Engl. J. Med. 322, 1829-1836 (1990).

7. Zlotogora J. \& Shapiro M.S. Polyglandular autoimmune syndrome type I among Iranian Jews. J. med. Cenet. 29, 824-826 (1992)

8. Norio R. Diseases of Finland and Scandinavia; Biocultural Aspects of Diseases (ed. Rothschild, H.R.) 359-415 (Academic Press, New York, 1981).

9. Syatnen, A.-C. et al. Convenient and Quantitative Determination of the Frequency of a Mutant Allele Using Solid-Phase Minisequencing: Application to Aspartylglucosaminurla in Finland. Genomics 12, 1-6 (1991).

10. Hastbacka, J. et al. Linkage disequilibrium mapping in isolated founder populations: diastrophic dysplasia in Finland. Nature Genet. 2, 204-211 (1992).

11. Lehesjoki, A.-E. et al. Localization of the EPM1 gene for progressive myoclonus epilepsy on chromosome 21: linkage disequilibrium allows high resolution mapping. Hum molec. Genet. 2, 1229-1234 (1993).

12. Aaltonen, J. ot al. Autoimmune Polyglandular Disease Type I: Exclusion Map Using Ampliflable Multiallelic Markers in a Microtiter Well Format. Eur. $J$. hum. Genet. 1, 164-171 (1993).

13. Lawrence, S., Collins, A., Keats, B.J., Hulten, M. \& Morton, N.E. Integration
$\mathrm{dN}) \pm 2 \alpha / \mathrm{d})$, with $\mathrm{N}$ equal to $10^{7} \sqrt{ }(1 / 25,000), \pi$ equal to $11 / 28$, and $\mathrm{d}$ equal to 0.0921 , we solved iteratively for $\alpha$, the frequency of recombination.

\section{Acknowledgements}

We are indebted to the APECED patients and their family members who participated in thisstudy. We thank Anne Vikman, Pekka Ahonen and Eric Lander for their help with this work. Grants from the Academy of Finland, University of Helsinki, Foundation for Pediatric Research, The Farmos Research Foundation, Clinical Research Institute of the Helsinki University Central Hospital are appreciated.

of gene maps: Chromosome21. Proc. natn. Acad. Sci. U.S.A. 90, 7210-7214 (1993).

14. Luria, S.E. \& Delbrück, M. Mutations of bacteria from virus sensitivity to virus resistance. Genet/cs 28, 491-511 (1943).

15. Carson, D.A. Genetic factors in the etiology and pathogenesis of autoimmunity. FASEB J. B, 2800-2805 (1992).

16. Hellsten, $E$ et al Refined Assignment of the Infantile Neuronal Ceroid Lipofuscinosis (INCL, CLN1) locus at 1p32: Incorporation of Linkage Disequilibrium in Multipoint Analysis. Genomics 16, 720-725 (1993).

17. Antonarakis, S.E. Human chromosome21: genomemapping and exploration, circa 1993. Trends Genet. 9, 142-148 (1993).

18. Gu, Z et al. Identification of a molecular defect in homocystinurla due to cystathionine beta-synthase deficiency. Am. J. hum. Genet. 49, 406A (1991).

19. Levanon, D., Danciger, E., Dafni, N. \& Groner, Y. Genomic clones of the human liver-type phosphofructokinase. Blochim. Blophys. res. Comm. 141, 374-386 (1986).

20. Arnaout, M., Dana, N., Gupta, S., Tenen, D. \& Fathallah, D. Point Mutations Impairing Cell Surface Expression of the Common $\beta$ Subunit (CD18) in a Patient with Leukocyte Adhesion Molecule (Leu-CAM) Deficiency. J. clin. Invest. 85, 977-989 (1990).

21. Hawkins, J.W. et al. Confirmation of assignment of the human alpha1-crystallin gene (CRYA1) to chromosome 21 with regional localization to q22.3. Hum. Genet. 76, 375-380 (1987).

22. Vandenplas, $\mathrm{S}$, et al. Blot hybridization analysis of genomic DNA. $J$. med. Genet. 21, 164-172 (1984).

23. Mullis, K.B \& Faloona, F.A. Specific synthesis of DNA in vitro via a polymerase-catalyzed chain reaction. Meth. Enzymol. 155, 335-350 (1987).

24. Lathrop, G.M. Lalouel, J.M., Julier, C. \& Ott, J. Strategies for multilocus linkage analysis in humans. Proc. natn. Acad. Sci. U.S.A. 81, 3443$3446(1984)$.

25. Conneally, P.M. et al. Report of the committee on methods of linkage analysis and reporting. Cytogenet. cell Genet. 40, 356-359 (1985). 